\title{
Improving results with first-stage palliation for hypoplastic left heart syndrome
}

Between January 1990 and February 1993, 73 patients underwent first-stage reconstruction for hypoplastic left heart syndrome at the University of Michigan Medical Center. During this period, surgical reconstruction remained essentially constant and consisted of a pulmonary artery-to-aorta anastomosis with allograft augmentation of the ascending, transverse, and proximal descending aorta, restriction of pulmonary blood flow with a polytetrafluoroethylene shunt from the innominate artery to the central pulmonary artery confluence, and atrial septectomy. Hospital survival was 62 of 73 patients, $85 \%$ ( $70 \%$ confidence limits: $80 \%$ to $89 \%$ ). These results stand in marked contrast to those obtained during the earlier years of our experience from 1986 to 1989 when only 21 of 50 patients $(42 \%, 70 \%$ confidence limits: $35 \%$ to $49 \%)$ survived $(p=0.001)$. Among the most recent group of patients, only 2 of 7 patients older than 1 month of age at operation survived, whereas 60 of $66(91 \%$, $70 \%$ confidence limits: $87 \%$ to $94 \%)$ patients younger than 1 month of age survived $(p=0.0001)$. Anatomic subtype and ascending aortic diameter were not predictive of survival. Actuarial survivals for those patients younger than 1 month of age at the first-stage operation, including hospital deaths and subsequent operative procedures, were $81 \%, 74 \%$, and $74 \%$ at 6 months, 1 year, and 2 years, respectively. These results indicate that survival for patients after first-stage reconstruction for hypoplastic left heart syndrome has significantly improved in recent years. Older age was a strong risk factor, with a hospital survival of $91 \%$ for those patients undergoing first-stage palliation within the first month of life. These data have important implications for the type of operative intervention and its timing. (J ThORAC CARdiovasc Surg 1994;107:934-40)

Mark D. Iannettoni, MD, ${ }^{\mathrm{a}}$ Edward L. Bove, MD, ${ }^{\mathrm{a}}$ Ralph S. Mosca, MD, ${ }^{\mathrm{a}}$

Flavian M. Lupinetti, MD, ${ }^{a}$ Parvin C. Dorostkar, MD, ${ }^{b}$ Achi Ludomirsky, MD,

Dennis C. Crowley, MD, Thomas J. Kulik, MD, and Amnon Rosenthal, MD,

Ann Arbor, Mich.

$P_{r}$ ago, hypoplastic left heart syndrome (HLHS) in neonates has recently been treated with dramatic successes with staged reconstructive and replacement therapies. ${ }^{1-5}$ Both

From the Section of Thoracic Surgery, Department of Surgery, ${ }^{\mathrm{a}}$ and the Division of Pediatric Cardiology, Department of Pediatrics, ${ }^{b}$ The University of Michigan Medical School, Ann Arbor, Mich.

Presented at the Sixty-fifth Scientific Session of the American Heart Association, New Orleans, La., Nov. 17, 1992.

Received for publication April 23, 1993.

Accepted for publication July 30, 1993.

Address for reprints: Edward L. Bove, MD, 1500 East Medical Center Dr., 2120 Taubman Center, Box 0344, Ann Arbor, MI 48109.

Copyright $\odot 1994$ by Mosby-Year Book, Inc.

$0022-5223 / 94 \$ 3.00+0 \quad 12 / 1 / 50468$ of these surgical options continue to have significant risk, however, and neither has emerged as clearly superior. The cumulative risk associated with multistaged reconstructive procedures and the ultimate limitations of a single ventricle physiology must be weighed against that imposed by the shortage of donor hearts, the time constraints resulting from the pathophysiology of medical palliation while awaiting transplantation, and the effects of long-term immunosuppression. For these reasons, we have adopted a policy of offering surgical treatment for neonates with HLHS by means of staged reconstruction eventually leading to repair by a Fontan procedure. Our initial results with this effort were published previously and included a high mortality. ${ }^{6}$ However, increasing experience has led to improved intraoperative techniques in addition to refinements in management both before and 
after operation. The purpose of this report is to review our more recent experience with surgical therapy for HLHS to assess the current results and the effect of multiple patient, procedural, and morphologic risk factors on outcome.

\section{Patients and methods}

Patient data. The entire experience with first-stage palliation of HLHS from January 1986 through February 1993 at C. S. Mott Children's Hospital, University of Michigan Medical Center, includes 123 patients. The results of the initial group of 50 patients who underwent operation between 1986 and 1989 were previously described. ${ }^{6}$ This current report presents a detailed analysis of the hospital mortality, late outcome, and risk factors for death for the latter group of 73 patients who were treated over the 3-year period between January 1990 and February 1993. All patients with the diagnosis of HLHS were offered palliation with a Norwood reconstructive procedure, and none were excluded on the basis of preoperative diagnostic studies, morphologic characteristics, condition, or age.

Each patient had a complete examination with Doppler echocardiography for noninvasive evaluation of cardiac anatomy. The echocardiographic diagnosis of HLHS was established if all three segments of the left ventricle were poorly developed. Patients with patent aortic or mitral valves were considered to have functional HLHS if at least two of the following preoperative echocardiographic criteria were met: (1) aortic anulus diameter of less than $5 \mathrm{~mm}$, (2) mitral anulus diameter of less than $8 \mathrm{~mm}$, and (3) a nonapex-forming left ventricle. Patients with complete atrioventricular septal defects were treated as having HLHS when less than one third of the common atrioventricular valve was committed to the left ventricle. Cardiac catheterization was occasionally performed to gain additional information, particularly in those patients with borderline left ventricular size, to assist in the decision-making process for the optimal method of treatment.

Surgical technique. The method of surgical repair has remained essentially constant over the time frame of this analysis and is, with some variations, the technique previously described by Pigott and associates. ${ }^{7}$ Cardiopulmonary bypass is established by cannulation of the proximal main pulmonary artery just distal to the pulmonary valve and the right atrial appendage. The branch right and left pulmonary arteries are occluded and systemic cooling is begun in preparation for circulatory arrest. After bypass is begun, the ascending, transverse, and proximal descending segments of the aorta are mobilized, and tourniquets are placed around each branch vessel. A large cryopreserved pulmonary allograft is trimmed to fashion a patch that will serve to augment the aorta and allow anastomosis to the proximal main pulmonary trunk. The circulation is arrested after a minimum cooling period of 20 minutes at a nasopharyngeal temperature of $20^{\circ} \mathrm{C}$ or less, and the branch vessels are occluded. The cannulas are removed, and the septum primum is completely excised. The main pulmonary trunk is then divided at the bifurcation, and the distal end is closed with a patch of allograft or polytetrafluoroethylene material to prevent narrowing of the left pulmonary artery. The ductal tissue is then completely excised from the undersurface of the aortic arch, and the resultant opening is extended at least 10 to $15 \mathrm{~mm}$ farther into the descending aorta. This incision is then extended proximally under the transverse arch and down the diminutive ascending aorta until the level of the previously divided main pulmonary trunk is reached. Great care must be taken here to avoid spiraling this incision. Augmentation of the aorta is then begun by attaching the ascending aorta to the main pulmonary artery with multiple interrupted monofilament sutures in the proximal corner near the coronary arteries. It is important to maintain a posterior orientation of the ascending aorta relative to the main pulmonary artery. The remainder of the aorta is attached to the pulmonary allograft incorporating the main pulmonary trunk proximally.

The heart is filled with saline solution to displace air, and the cannulas are replaced to begin bypass and commence systemic rewarming. An appropriately sized polytetrafluoroethylene conduit is anastomosed from the innominate artery to the central pulmonary artery during the rewarming. A $4 \mathrm{~mm}$ conduit is used for patients weighing more than 3.5 to $4 \mathrm{~kg}$, and a $3.5 \mathrm{~mm}$ conduit is used for patients who weigh less than that. On a few occasions, for very small patients, $3 \mathrm{~mm}$ grafts have been used. The distal end of the shunt is placed centrally, close to the divided end of the ductus rather than onto the right pulmonary artery itself. This placement has resulted in better growth of the left pulmonary artery, avoiding the asymmetric growth that was frequently seen when the distal end of the shunt was placed to the right pulmonary artery (Fig. 1).

Postoperative management. After bypass is discontinued, a right atrial monitoring catheter is placed to measure central venous pressure, and the administration of dobutamine at 5 to $10 \mu \mathrm{g} / \mathrm{kg}$ per minute is begun. Ventilation with an inspired oxygen concentration of $100 \%$ to achieve a carbon dioxide tension of approximately $35 \mathrm{~mm} \mathrm{Hg}$ is initiated and rapidly adjusted depending on the systemic saturation and the hemodynamics. If hypotension with systemic oxygen saturations in excess of $80 \%$ to $85 \%$ are noted, the inspired oxygen concentration and minute ventilation are rapidly decreased. Dopamine is generally substituted for dobutamine to stimulate an increase in pulmonary vascular resistance and decrease pulmonary blood flow. The opposite maneuvers are used if the systemic oxygen saturation is less than $70 \%$ to $75 \%$.

The postoperative management is aimed at maintaining the delicate balance between the systemic and pulmonary vascular resistances and avoiding shifts of either one relative to the other. Ideally, the systemic arterial oxygen saturation should be maintained at $75 \%$ to $80 \%$, which generally indicates that the pulmonary-to-systemic blood flow ratio is less than 1.5. A continuous infusion of fentanyl, begun during the operation and maintained for 24 to 48 hours, is used to blunt the stress response of the pulmonary vasculature and prevent important fluctuations in systemic oxygen saturation that may be provoked with noxious stimuli, such as suctioning. ${ }^{8}$

Data collection and statistics. The medical records, operative reports and echocardiographic results for all patients were reviewed. Subsequent operative procedures were noted. Survival probability was determined by Kaplan-Meier methods. Follow-up was complete for all patients. The probability for secondand third-stage operations was determined by actuarial survival curves. Univariate and multivariate analyses were used to determine significant risk factors, alone or in combination, that could predict outcome. The following risk factors were evaluated: age, weight, year of operation (1986-89 versus 1990-93), 


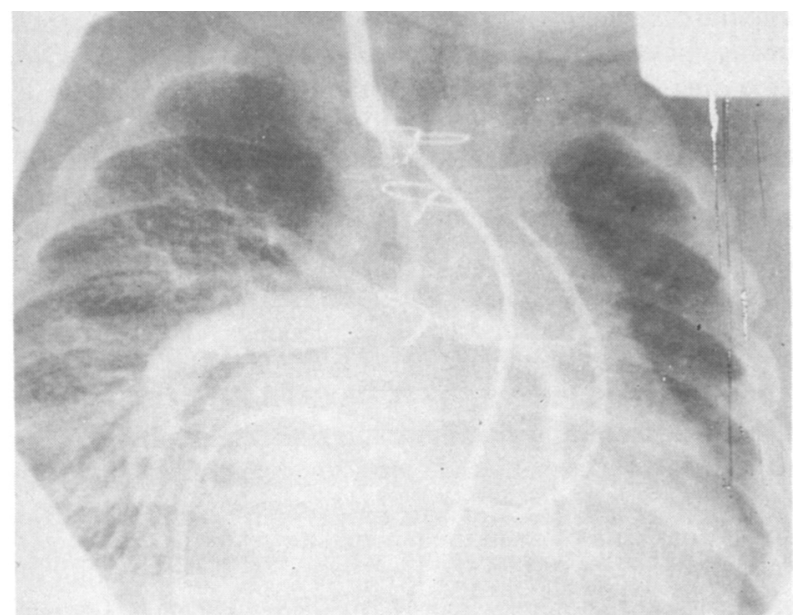

A

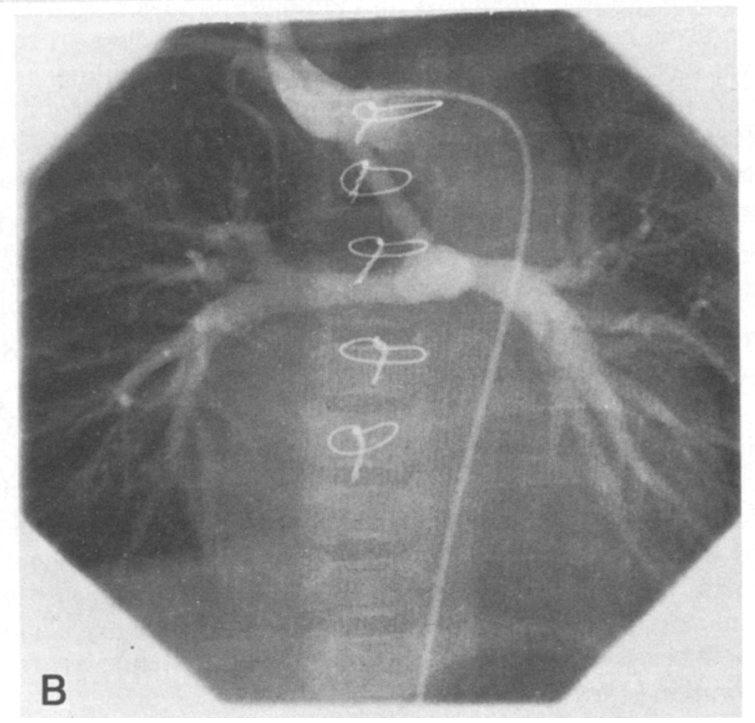

Fig. 1. A, Angiogram showing asymmetric growth of the pulmonary arteries with significant hypoplasia of the left pulmonary artery in a patient with the distal end of the shunt placed to the right pulmonary artery. B, Improved position for the distal end of the shunt is shown; it is placed to the central pulmonary artery confluence adjacent to the ligated ductus arteriosus. Symmetric growth of both pulmonary arteries is shown.

initial oxygen tension and $\mathrm{pH}$ at the time of admission to the hospital, morphologic subgroup, ascending aorta size, shunt size, and circulatory arrest time.

\section{Results}

Preoperative condition. There were 41 boys and 32 girls. The majority of patients were younger than 2 weeks of age at the time of operation (median age 7 days, range 1 to 210 days). Only seven patients were older than 1 month of age. The average weight at the time of opera-

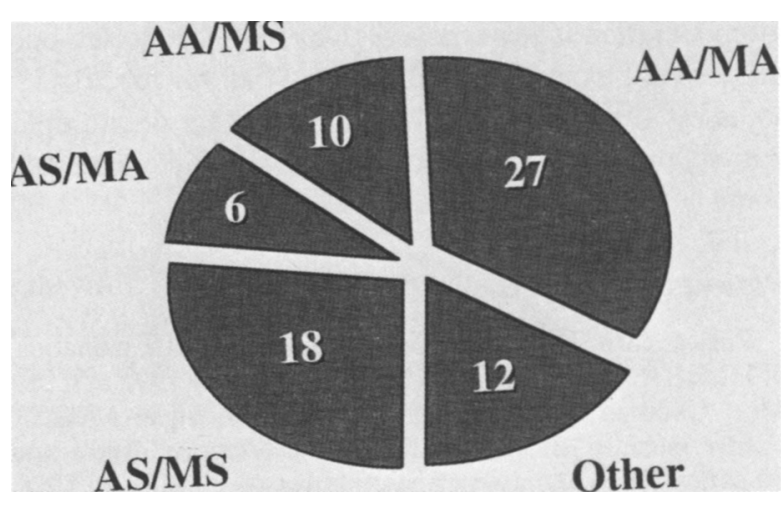

Fig. 2. Morphologic subgroups for all patients undergoing first-stage reconstruction $(n=73)$. $A A$, Aortic atresia, $M A$, mitral atresia; $A S$, aortic stenosis; $M S$, mitral stenosis.

tion was $3.4 \mathrm{~kg}$ (range 1.5 to $5.1 \mathrm{~kg}$ ). Preoperative inotropic support was used in 31 patients, classified as minimal in 18 patients and moderate-to-high in 13. Sixty-two patients underwent mechanical ventilation of the lungs before operation, and 68 patients were receiving an infusion of prostaglandin $E_{1}$. The initial oxygen tension and $\mathrm{pH}$ were evaluated at the time of diagnosis and corrected with appropriate ventilatory management, sodium bicarbonate, or both as needed. The mean oxygen tension at the time of diagnosis was $47 \pm 9 \mathrm{~mm} \mathrm{Hg}$ (range 26 to $74 \mathrm{~mm}$ $\mathrm{Hg}$ ). The initial $\mathrm{pH}$ at the time of admission to our hospital was less than 7.40 in only five patients. Many patients, however, were resuscitated at the referring institution before transfer.

Morphologic characteristics. There were 27 patients (37\%) with aortic and mitral atresia, 10 patients $(14 \%)$ with aortic atresia and mitral stenosis, 6 patients $(8 \%)$ with aortic stenosis and mitral atresia, and 18 patients (25\%) with aortic and mitral stenosis (Fig. 2). The remaining 12 patients $(16 \%)$ had complete atrioventricular canal defects with a hypoplastic left ventricle. The mean diameter of the ascending aorta as evaluated by echocardiography was $3.4 \pm 1.7 \mathrm{~mm}$ (range 0.5 to 10 $\mathrm{mm}$ ). The diameter of the ascending aorta was less than $3 \mathrm{~mm}$ in $56 \%$ of the patients and was less than $2 \mathrm{~mm}$ in $30 \%$ (Fig. 3). A left superior vena cava was present in 12 patients. Right ventricular function was determined to be good in 63 patients, fair in eight patients, and poor in two patients.

Surgical data. The mean circulatory arrest time was $50 \pm 9$ minutes (range 25 to 74 minutes). A $4.0 \mathrm{~mm}$ shunt was used in 42 patients, a $3.5 \mathrm{~mm}$ shunt in 18 patients, and a $3.0 \mathrm{~mm}$ shunt in 10 patients. In three large patients, a $5.0 \mathrm{~mm}$ shunt was used in two and a $6.0 \mathrm{~mm}$ shunt in one (Fig. 4). 


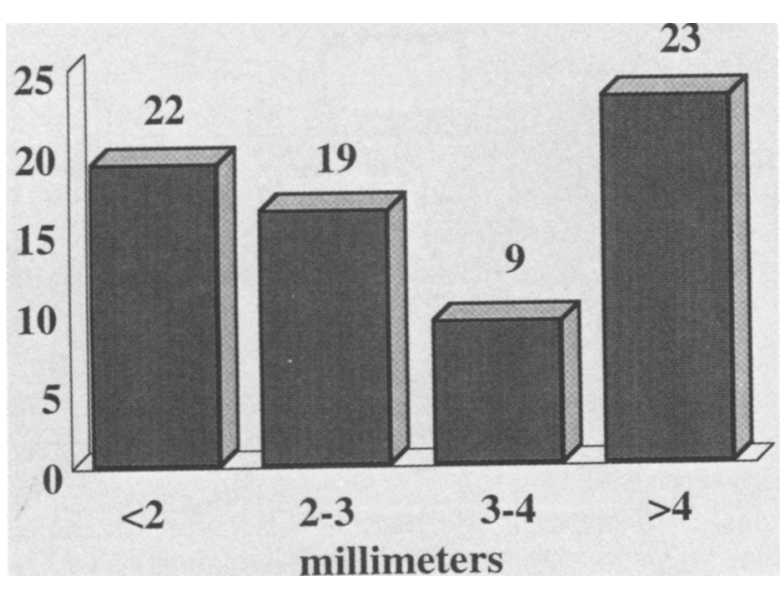

Fig. 3. Distribution of ascending aortic diameters as determined by preoperative echocardiography $(n=73)$.

Table I. Hospital survival after first-stage reconstruction for HLHS according to year

\begin{tabular}{ccccc}
\hline \multicolumn{1}{c}{ Year } & $\begin{array}{c}\text { No. of } \\
\text { patients }\end{array}$ & Survival & $\begin{array}{c}70 \% \\
C L\end{array}$ & $\begin{array}{c}\mathrm{p} \\
\text { Value }\end{array}$ \\
\hline $1986-89$ & 50 & $21(42 \%)$ & $35 \%-49 \%$ & \\
$1990-93^{*}$ & 73 & $62(85 \%)$ & $80 \%-89 \%$ & 0.001 \\
\hline
\end{tabular}

*Data up to February 1993.

Survival. Between January 1990 and February 1993, 62 of 73 patients $(85 \%, 70 \%$ confidence limit [CL]: $80 \%$ to $89 \%$ ) undergoing first-stage palliation with a Norwood procedure survived to hospital discharge. This represents a significant improvement when compared with the results achieved between 1986 and 1989 (Table I). ${ }^{6}$ During that earlier time frame, only 21 of 50 patients $(42 \%$, $70 \% \mathrm{CL}: 35 \%$ to $49 \%$ ) survived ( $p=0.001)$. In addition to the year of operation, the age at operation had an important effect on outcome (Table II) with 60 of 66 patients $(91 \%, 70 \% \mathrm{CL}: 87 \%$ to $94 \%)$ younger than 1 month surviving compared with 2 of 7 patients $(29 \%, 70 \%$ CL: $15 \%$ to $48 \%$ ) who were older than 1 month of age $(p=0.001)$. Although those patients older than 1 month of age represent only $10 \%$ of the patient population, they account for $45 \%$ of the hospital deaths. Most of these older patients died of pulmonary hypertensive crises. Actuarial survival for the entire group of 73 patients, including hospital deaths and subsequent operative procedures, was $76 \%$ at 6 months, $69 \%$ at 1 year, and $69 \%$ at 2 years. Actuarial survival for the 66 patients younger than 1 month at first-stage operation was $81 \%, 74 \%$, and $74 \%$ at 6 months, 1 year, and 2 years, respectively (Fig. 5).

There were 19 deaths in the series, 18 of which occurred

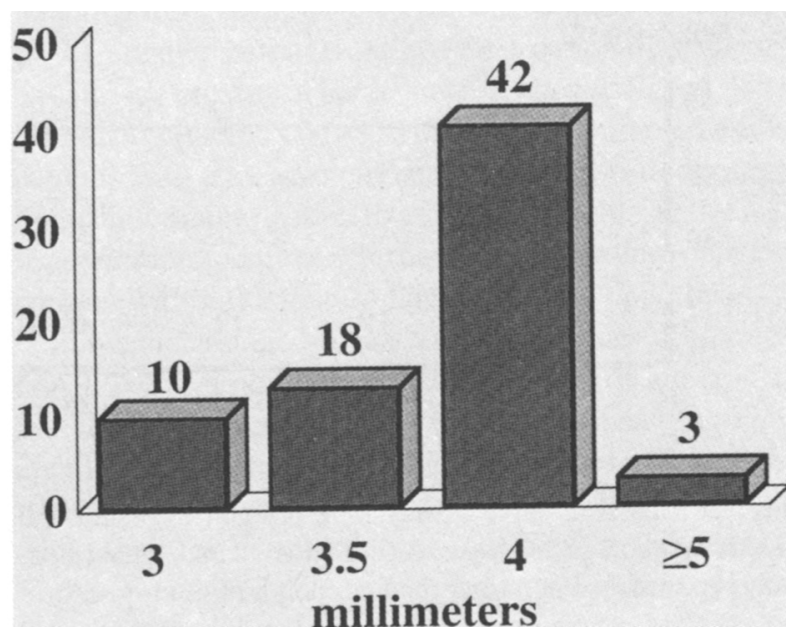

Fig. 4. The distribution of shunt diameters placed at firststage reconstruction $(n=73)$.

Table II. Hospital survival after first-stage reconstruction for HLHS according to age

\begin{tabular}{ccrcc}
\hline Age & No. of & & $70 \%$ & $\mathrm{p}$ \\
patients & Survival & \multicolumn{1}{c}{$C L$} & Value \\
\hline$<1$ month & 66 & $60(91 \%)$ & $87 \%-94 \%$ & \\
$>1$ month & 7 & $2(29 \%)$ & $15 \%-48 \%$ & 0.001 \\
\hline
\end{tabular}

after first-stage palliation for HLHS and one after the bidirectional Glenn procedure. Eleven patients died early (less than 30 days), and eight died late. There were 14 deaths in those patients younger than 1 month of age at the time of first-stage palliation, with six of these being early and eight being late. In those patients older than 1 month of age at initial operation, there were five deaths among seven patients, all of which were early. In the first 48 hours after operation, $47 \%$ of the deaths in the series occurred. The late deaths resulted from sepsis in four, pulmonary vascular disease in two, severe right ventricular dysfunction in one, and unknown causes in one patient.

Risk factor analysis. Risk factor analysis was performed on all variables with univariate and, when appropriate, multivariate techniques. Risk stratification demonstrated only two significant preoperative risk factors as predictors of death: year of operation (1986-89 versus 1990-93) and age at operation (older than 1 month) as noted previously. The weight, specific morphologic subgroup, ascending aortic size, shunt size, initial oxygen tension or $\mathrm{pH}$ at the time of admission to the hospital, or circulatory arrest time were not found to be predictors of mortality.

Late follow-up. Among the group of 62 hospital survivors, there were eight late deaths. The follow-up of the 


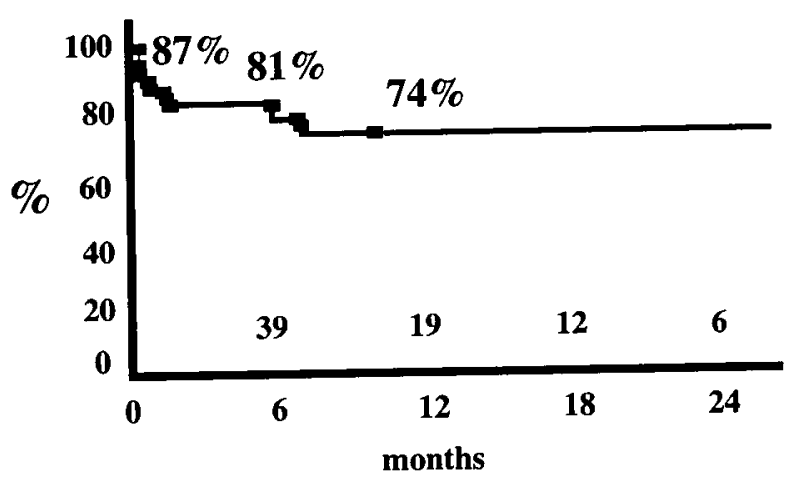

Fig. 5. Actuarial survival, including hospital mortality and deaths at subsequent stages, for all patients undergoing firststage reconstruction during the first month of life $(n=66)$.

hospital survivors is shown in Fig. 6. Of the 54 remaining patients, 37 have undergone the bidirectional Glenn procedure and 17 await second-stage repair. There was one hospital death after the bidirectional Glenn procedure with no late deaths. Of the 36 survivors after second-stage repair, 14 patients underwent the Fontan procedure with no deaths.

\section{Discussion}

The outlook for infants born with HLHS has dramatically improved in the past few years. Increasing experience with both reconstructive and replacement techniques has resulted in significant improvements in the overall quantity and quality of survival. ${ }^{1-5}$ Because neither approach has been proved to be clearly superior to the other, our group has chosen to adopt a policy of offering staged reconstruction to gain enough experience to properly assess its limitations. Although our earlier efforts were associated with a high mortality, sufficient improvement was made with increasing experience to justify continuing this policy.

Previous attempts to identify perioperative patient, morphologic, and procedural risk factors for death have not been uniformly defined. ${ }^{9}$ In this study, only year of operation and age beyond 1 month were found to be significant predictors of increased mortality. Importantly, morphologic subgroup and ascending aortic size were not associated with an increased risk, as has been suggested by others..$^{10}$ Although those patients with aortic diameters smaller than $3 \mathrm{~mm}$ pose increased technical difficulties during reconstruction, this potential risk has been neutralized by the operative techniques used in this series. There appears to be no inherent reason why patients with diminutive ascending aortas cannot achieve the same results as those with larger aortas. Furthermore, no

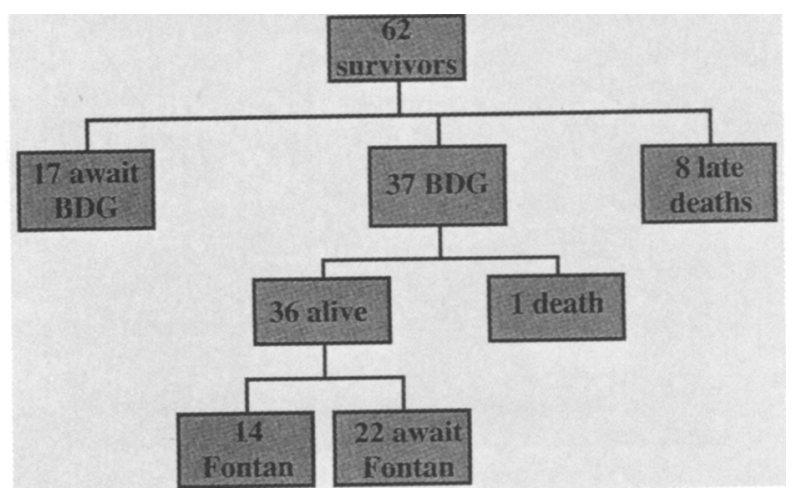

Fig. 6. The current status of all hospital survivors (62 of 73) after first-stage reconstruction. $B D G$, Bidirectional Glenn shunt.

increased risk was found among those patients with aortic atresia and mitral stenosis, a morphologic subgroup that has been noted by Jonas and associate ${ }^{10}$ to be a weak predictor of early mortality. Sauer and associates ${ }^{11}$ found an increased prevalence of coronary artery anomalies in this subgroup, most notably persistent ventriculocoronary communications, which might result in diminished right ventricular myocardial blood flow. The risk factor analysis also demonstrated that poor preoperative condition was not associated with increased risk. Many patients in this experience had acidosis, poor perfusion, and tricuspid valve regurgitation as a result of low cardiac output and right ventricular dysfunction at the time they were examined in the initially referring hospital. ${ }^{12}$ Preoperative resuscitation with inotropic agents, mechanical ventilation to regulate pulmonary blood flow, and treatment of associated conditions were usually successful in reversing right ventricular dysfunction. Additional support for these findings was reported by Helton ${ }^{9}$ and Murdison ${ }^{13}$ and their associates.

The increased risk found among those patients in this series older than 1 month of age has important implications for treatment, particularly for those institutions choosing to perform a Norwood procedure only if a suitable donor heart does not become available for transplantation after a certain waiting period. Among patients younger than 1 month of age at operation, $91 \%$ survived to hospital discharge. In contrast, only $29 \%$ of patients older than 1 month survived. The majority of the older patients died of pulmonary hypertensive crises that resulted in severe hypoxemia, a process that was accentuated by the limited degree of pulmonary blood flow imposed by a restrictive systemic-to-pulmonary artery shunt. It is our belief that optimal early results are obtained when a small shunt is used to avoid a large ventricular volume 
overload and its attendant right ventricular dysfunction. Additionally, a large shunt results in low diastolic blood pressures, which may further aggravate ventricular failure because of decreased myocardial blood flow. Although we were able to achieve success in older patients when larger diameter shunts were used ( $\geq 5 \mathrm{~mm})$, the large volume overload and low diastolic pressure resulted in poor ventricular function and significant congestive heart failure during those periods of time when pulmonary vascular resistance was low. In these patients, an early second-stage bidirectional Glenn procedure may be necessary after the early lability of the pulmonary vasculature has resolved. Alternatively, insertion of smaller shunts with pharmacologic control of the pulmonary vascular resistance with the use of newer agents, such as nitric oxide, may provide optimal early regulation of pulmonary blood flow until such time as the pulmonary vascular resistance remains low.

The use of smaller shunts at the initial procedure in addition to the placement of the distal anastomosis in a central position adjacent to the ligated ductus arteriosus has generally resulted in lower systemic oxygen saturations during the early recovery period. This benefit has simplified the early postoperative management and has obviated the need to use significant degrees of hypoventilation or increased concentrations of carbon dioxide in the inspired gas mixture to control excessive pulmonary blood flow in the majority of our patients, as suggested by Jobes and associates. ${ }^{14}$ Relatively minor alterations in minute ventilation, resulting in arterial carbon dioxide tensions of 35 to $45 \mathrm{~mm} \mathrm{Hg}$, have generally been sufficient to maintain acceptable hemodynamics when systemic arterial oxygen saturation was in excess of $85 \%$. The optimal systemic oxygen saturation of $75 \%$ to $80 \%$ was generally maintained by adjusting the inspired oxygen concentration alone. The administration of dopamine in doses of 8 to $10 \mu \mathrm{g} / \mathrm{kg}$ per minute may serve to increase pulmonary vascular resistance and improve the hemodynamics in those patients with excessive pulmonary blood flow. Although these smaller shunts have resulted in improved early hemodynamics, some patients have required second-stage reconstruction with a bidirectional Glenn procedure by 3 or 4 months of age when significant hypoxemia occurs as the shunt is outgrown. However, our experience with the second-stage procedure has shown that the procedure may be successfully performed as early as 1 month of age. ${ }^{15}$ This knowledge has encouraged us to continue to restrict pulmonary blood flow with small shunts at the first stage operation. Despite this experience, however, a more precise determination of the optimal shunt size for each patient is desirable. We have followed a general rule of inserting $4 \mathrm{~mm}$ conduits only in patients weighing more than 3.5 to $4 \mathrm{~kg}$ and 3 to $3.5 \mathrm{~mm}$ conduits in the smaller patients. Although occasional problems have arisen because of inadequate pulmonary blood flow, excessive pulmonary flow has been more common. Of five patients with excessive pulmonary flow, two underwent immediate shunt revision to a smaller size during the initial operation and three returned to the operating room for revision within the first 24 hours after the operation.

Subsequent staged reconstruction includes a bidirectional Glenn procedure at 4 to 6 months of age and a Fontan procedure at 18 to 24 months. ${ }^{15,16}$ This approached is based on the objective of allowing the ventricle to gradually adjust to a diminished volume load and allow time for a reduction in muscle mass before the Fontan procedure. ${ }^{15,17-19}$ Although studies have shown that the bidirectional Glenn procedure causes little acute reduction of ventricular mass, there is evidence of a reduction in right ventricular work. ${ }^{17}$ Perhaps late survival has improved as a result of this staging. Survival after the bidirectional Glenn procedure has been excellent with only one hospital death and no late deaths. No deaths occurred after the Fontan procedure.

These data suggest that staged reconstruction can be recommended for all patients with HLHS regardless of anatomic subtype or aortic size with an equal likelihood of success. Because older age so adversely affected survival, however, treatment strategies that emphasize cardiac transplantation should be examined in this context. In particular, prolonged waiting for a transplant donor may make patients less suitable for staged reconstruction if a heart does not become available within the first month of life.

\section{REFER ENCES}

1. Norwood WI Jr. Hypoplastic left heart syndrome. Ann Thorac Surg 52;1991:688-95.

2. Jonas RA, Lang P, Hansen D, Hickey, Castaneda AR. First-stage palliation for hypoplastic left heart syndrome.

3. Bailey LL, Assaad AN, Trimm RF, Nehlsen-Cannarella SL, Kanakriyeh MS, Haas GS. Orthotopic transplantation during early infancy as therapy for incurable congenital heart disease. Ann Thorac Surg 1988;208:279-86.

4. Backer CL, Zales VR, Harrison HL, Idriss FS, Benson DW Jr, Mavroudis C. Intermediate-term results of infant orthotopic cardiac transplantation from two centers. J THORAC CARDIOVASC SURG 1991;101:826-32.

5. Bove EL. Transplantation after first-stage reconstruction for hypoplastic left heart syndrome. Ann Thorac Surg 1991;52:701-7.

6. Meliones JN, Snider R, Bove EL, Rosenthal A, Rosen DA. Longitudinal results after first-stage palliation for hypoplastic left heart syndrome. Circulation 1990;82(Suppl):IV151-6. 
7. Pigott JD, Murphy JD, Barber G, Norwood WI. Palliative reconstructive surgery for hypoplastic left heart syndrome. Ann Thorac Surg 1988;45:122-8.

8. Hansen DD, Hickey PR. Anesthesia for hypoplastic left heart syndrome. Anesth Analg 1986;65:127-32.

9. Helton JG, Aglira BA, Chin AJ, Murphy JD, Pigott JD, Norwood WI. Analysis of potential anatomic or physiologic determinants of outcome of palliative surgery for hypoplastic left heart syndrome. Circulation 1986;74(Sup$\mathrm{pl}): \mathrm{I} 70-6$.

10. Jonas RA, Hansen D, Cook N, Wessel D. Anatomic subtype of hypoplastic left heart syndrome influences survival after palliative reconstruction. Proceedings of the seventysecond Annual Meeting of The American Association of Thoracic Surgery April 26-29, 1992; Los Angeles, Calif.

11. Sauer U, Gittenberger-de Groot AC, Geishauser M, Babic $\mathrm{R}$, Buhlmeyer K. Coronary arteries in the hypoplastic left heart syndrome: Histopathologic and histometrical studies and implications for surgery. Circulation 1989;80 (Supp1):I168-76.

12. . Moodie DS, Gill CC, Sterba R, Stewart R, Ratliff NB. The hypoplastic left heart syndrome: evidence of preoperative myocardial and hepatic infarction in spite of prostaglandin therapy. Ann Thorac Surg 1986;42:307-11.
13. Murdison KA, Baffa JM, Farrell PE Jr, et al. Hypoplastic left heart syndrome. Circulation 1990;82(Suppl):IV199207.

14. Jobes DR, Nicolson SC, Steven JM, Miller M, Jacobs ML, Norwood WI. Carbon dioxide prevents pulmonary overcirculation in hypoplastic left heart syndrome. Ann Thor Surg 1992;54:150-1.

15. Pridjian AK, Mendelsohn A, Lupinetti FM, Beekman RH III, Dick M II, Serwer G, Bove EL. Usefulness of the bidirectional Glenn procedure as a staged reconstruction for the functional single ventricle. Am J Cardiol 1993;71:959-62.

16. Norwood WI Jr, Jacobs ML, Murphy JD. Fontan procedure for hypoplastic left heart syndrome. Ann Thorac Surg 1992;54:1025-30.

17. Gewillig M, Daenen W, Aubert A, Van der Hauwaert L. Abolishment of chronic volume overload. Circulation 1992;86(Suppl):II93-9.

18. Lamberti J. Palliation of univentricular heart without increasing ventricular work [Editorial]. Ann Thorac Surg 1991;51:882-3.

19. Douville EC, Sade RM, Fyfe DA. Hemi-Fontan operation in surgery for single ventricle: a preliminary report. Ann Thorac Surg 1991;51:893-900. 